\title{
Modeling and Simulation of Vibration Signatures in Propulsion Subsystems
}

\author{
Nancy Lybeck, Brogan Morton, \\ Sean Marble \\ Sentient Corporation \\ 850 Energy Drive \\ Idaho Falls, ID 83401 \\ (208)522-8560 \\ nlybeck@sentientscience.com
}

\author{
Andrew Hess \\ Air System PHM Lead \\ JSF Program Office \\ 1213 Jefferson Davis Highway \\ Arlington, VA 22202-4304
}

\author{
John Kelly \\ Electronics Engineer \\ NAWCAD Bldg 2133 \\ Patuxent River, MD 20670 \\ (301) 757-4368
}

\begin{abstract}
A fundamental problem in the development and validation of PHM technologies is the general shortage of realistic fault signature data. While healthy signatures can be obtained from operational systems, faults are relatively rare and difficult to observe. The PHM community must rely primarily on bench level seeded fault test data collected under a limited set of conditions. To augment physical data, a modeling and simulation toolset is being developed for the vibration signatures of faulted components in propulsion subsystems such as gearboxes. The toolset includes sophisticated dynamic models of vibration forcing for common rotating components such as bearings and gears based on detailed analysis of their physical interactions, including the effects of faults such as bearing spalling or gear tooth cracks. The response of the overall system, and thus the vibration signal seen at a particular sensor location can then be predicted using either FEA or a transfer function analysis of actual hardware. The purpose of the toolset is to leverage seeded fault test data (for example to study operating conditions or fault types that were not tested), improve fault diagnosability through optimal sensor placement, and enhance development, testing, and validation of diagnostic systems. Several examples are presented comparing simulated vibration signals to actual test data. ${ }^{1,2}$
\end{abstract}

\section{TABLE OF CONTENTS}

1. INTRODUCTION ............................................................1

2. PREVIOUS WORK …………………………………….....2

3. VIBRATION DIAGNOSTICS......................................2

4. PHYSICS-BASEd MOdELS .........................................3

5. COMPARISON WITH EXPERIMENTAL RESULTS....4

6. APPLICATIONS............................................................6

7. CoNCLUSIONS ................................................................7

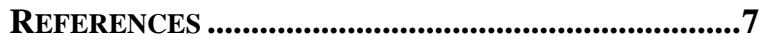

ACKNOWLEDGMENTS ....................................................

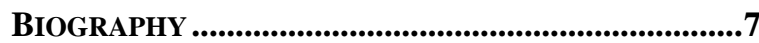

\footnotetext{
${ }^{1}$ 0-7803-9546-8/06/\$20.00@ 2006 IEEE

2 IEEEAC paper \#1351, Version 1
}

\section{INTRODUCTION}

Vibration is the most commonly used parameter to monitor system health in the aerospace industry. The interpretation of this data is often difficult, even for the most experienced technicians. Development and calibration of diagnostic algorithms requires access to quality data for faulted systems.

The development and maturation of PHM technologies is limited by the availability of fault signature data covering the full range of system operating conditions. Data for healthy systems is often readily available, but fault data is much more difficult to obtain. Seeded fault tests are time consuming, expensive, and are not always representative of actual operation. High-fidelity fault signature simulation models are needed to supplement physical testing and field data.

In cases where operational data is not yet available or is incomplete, realistic vibration models could provide an effective tool for PHM development and maturation. A complete tool set modeling the signature vibrations emitted by faulted bearings, gears, and shaft imbalances, has a multitude of practical uses, such as determining the optimal locations for accelerometers in a conceptual design based on FEA data, or in an existing system based on ping test data. The tool set could also be used to test PHM systems onwing by feeding them simulated vibration data and checking for the appropriate indication to rule out the possibility of a malfunction or false alarm. The fault vibration models might even be integrated into the built-in-self-test function of PHM systems.

Sentient Corporation has developed a fault vibration simulator (FVS), a modeling and simulation toolset for the vibration signatures of faulted bearings. The model is extensible to other faulted rotating machinery, such as gears with tooth pitting, tooth root cracks, or shaft imbalances. Experimental results are presented indicating the high level of agreement between simulated bearing vibration signals and actual test data. Ongoing work includes optimizing the simulator for bearings, and calibrating the models for gears. 


\section{Report Documentation Page}

Form Approved

OMB No. 0704-0188

Public reporting burden for the collection of information is estimated to average 1 hour per response, including the time for reviewing instructions, searching existing data sources, gathering and maintaining the data needed, and completing and reviewing the collection of information. Send comments regarding this burden estimate or any other aspect of this collection of information,

including suggestions for reducing this burden, to Washington Headquarters Services, Directorate for Information Operations and Reports, 1215 Jefferson Davis Highway, Suite 1204, Arlington

VA 22202-4302. Respondents should be aware that notwithstanding any other provision of law, no person shall be subject to a penalty for failing to comply with a collection of information if it

does not display a currently valid OMB control number.

\begin{tabular}{|l|l|l}
\hline 1. REPORT DATE & 2. REPORT TYPE & 3. DATES COVERED \\
\hline N1 OCT 2006 & N/A & 5a. CONTRACT NUMBER \\
\cline { 2 - 2 } $\begin{array}{l}\text { 4. TITLE AND SUBTITLE } \\
\text { Subsystems }\end{array}$ & 5b. GRANT NUMBER \\
\cline { 2 - 3 } & 5c. PROGRAM ELEMENT NUMBER \\
\hline 6. AUTHOR(S) & 5d. PROJECT NUMBER \\
\cline { 2 - 2 } & 5e. TASK NUMBER \\
\cline { 2 - 3 } & 5f. WORK UNIT NUMBER \\
\hline 7. PERFORMING ORGANIZATION NAME(S) AND ADDRESS(ES) & $\begin{array}{l}\text { 8. PERFORMING ORGANIZATION } \\
\text { REPORT NUMBER }\end{array}$ \\
\hline 9. SPONSORING/MONITORING AGENCY NAME(S) AND ADDRESS(ES) & 10. SPONSOR/MONITOR'S ACRONYM(S) \\
\cline { 2 - 3 } & $\begin{array}{l}\text { 11. SPONSOR/MONITOR'S REPORT } \\
\text { NUMBER(S) }\end{array}$
\end{tabular}

12. DISTRIBUTION/AVAILABILITY STATEMENT

Approved for public release, distribution unlimited

13. SUPPLEMENTARY NOTES

The original document contains color images.

14. ABSTRACT

15. SUBJECT TERMS

16. SECURITY CLASSIFICATION OF:

a. REPORT unclassified b. ABSTRACT unclassified c. THIS PAGE unclassified
17. LIMITATION OF ABSTRACT

$\mathbf{U U}$

\begin{tabular}{c|l}
$\begin{array}{c}\text { 18. NUMBER } \\
\text { OF PAGES } \\
\mathbf{8}\end{array}$ & 19a. NAME OF \\
&
\end{tabular}




\section{Previous WorK}

Figure 1 shows that generation of vibration from a bearing or gear can be broken into three stages. A forcing function is generated by the impact of a fault with bearing components, or by the meshing of teeth in gears. Knowledge of the forcing function could provide an indication of the severity of the fault, but direct measurement of the forcing function is not possible. The vibration must pass through the housing to the sensor location, where the output is measured. The frequency response indicates how the vibration is transmitted through the housing. must be used to account for the transmission of the gear meshing vibrations to the sensor.

McFadden and Smith's model architecture provides a modular, physics-based method for modeling the vibration response of bearings and gears. The authors have taken this model and extended it to the more general case.

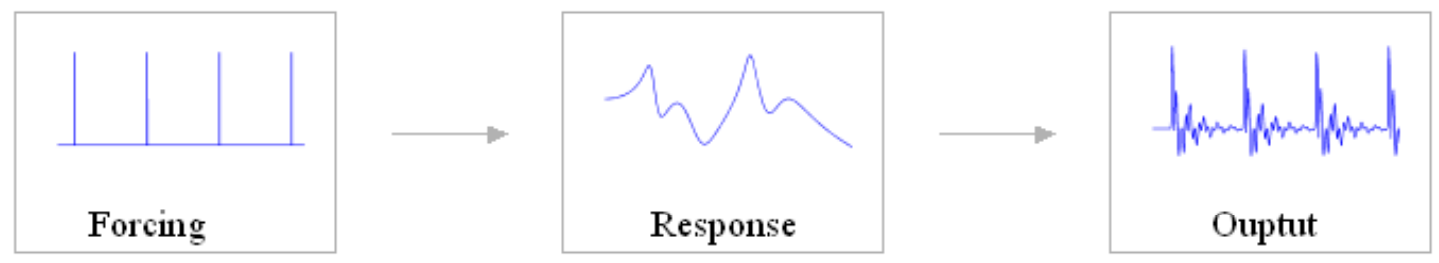

Figure 1 - Stages of vibration generation

Many scientists have researched the effects of gear and bearing defects on the vibration emitted by the component. A large percentage of this work has focused on developing signal processing techniques to detect and isolate faults. A relatively small number of papers have described a physicsbased model of the fault signature vibration.

McFadden and Smith [1] undertook the task of explaining the pattern of spectral lines seen in the vibrations emitted by faulted rolling element bearings under a radial load. The model they presented used a periodically repeated impulse function, $\delta(t)$, to model the vibration produced by an impact of a defect with a different bearing surface. The amplitude of the impulses gives an indication of the severity of the fault.

McFadden and Smith accounted for the load variations due to radial loading by modulating the amplitude of the impulse train. They employed a transfer function to emulate the transmission of the vibrations from the bearing to the sensor location.

In healthy gears, there is a forcing created by the impacts from teeth meshing. Local tooth defects such as pitting or fatigue cracks change the stiffness of the tooth, affecting the forcing vibration when the faulty tooth is engaged. McFadden [2] demonstrated that phase and amplitude modulation can provide an early warning of gear defects. He also presented an equation for the meshing vibration produced by local gear defects. As with bearings, the vibration is sensed on the housing. Thus transfer functions

\section{VibRATION Diagnostics}

When a bearing is faulted, the signature vibration is changed by the impacts produced by rolling elements passing over the fault. For a bearing undergoing rotation at a known, constant speed, the frequency of these impacts can be predicted given knowledge of the geometry of the bearing and the type of fault [3].

There are four fundamental frequencies that are of interest in bearing fault detection. The BPFO is the frequency with which a ball passes the trailing edge of a fault on the outer race. The BPFI is the frequency with which a ball passes the trailing edge of a fault on the inner race. The BFF is the frequency at which a fault on a ball passes both the outer and inner race (which is twice the ball speed). Finally, the FTF is the rotational speed of the ball/cage train with respect to the outer race.

The complexity of sensed vibration signals for bearings and gearboxes makes interpretation of the time series or spectral plots a challenging task. Thus statistical methods are traditionally used to provide thresholds indicating an impending failure [4]. The root mean square (RMS) of the data provides an indication of the overall signal energy. Kurtosis, the fourth statistical moment of the data, is a measure of the "spikiness" of the data. The Crest Factor is the ratio of the peak vibration level to the RMS, and is useful in detecting changes due to impulsive vibration forces. Enveloping is a signal processing tool that focuses on amplitude modulation, and is often useful for early detection of bearing faults. 
The above techniques are typically used on the raw vibration signal. The only preprocessing required is scaling using the calibration constant for the accelerometer. Time synchronous averaging (TSA) is a preprocessing technique that is used to remove non-stationary noise from a repetitive signal. The data is broken into segments representing one repetition (e.g., 1 revolution of a gear), and the segments are then averaged. Even slight variations in speed can create difficulties in the averaging process, leaving some segments shorter than others. Interpolation is used to account for this.

These commonly used diagnostic techniques provide a basis for judging the performance of the vibration simulator. Results presented in Section 5 use RMS, Kurtosis, and Crest Factor to compare simulated and experimental data.

\section{Physics-Based Models}

\subsection{Forcing Models}

Physics-based models were developed for the forcing vibration emitted by faulted rotating components. The dynamic forces in the bearing given geometry, speed, and load are calculated. The effects of ball-race impacts and modulated radial/axial forces are included in the model. The signal characteristics relevant to diagnostics can be modeled accurately.

Healthy bearings emit vibrations that are low-level broadband noise. For a bearing that is spalled, there are periodic impacts created when a ball passes the spall. The vibration spectrum indicates the presence and type of a fault, such as an outer race fault, an inner race fault, or a ball fault. A shaft imbalance causes the force to be modulated at the rotation rate.

Healthy gears emit vibrations at the gear meshing frequency. Spectrum analysis of gears with local tooth defects often shows modulation sidebands around the gear meshing frequency and its harmonics. However, even healthy gears often show modulation sidebands due to inherent meshing errors. This complicates the spectral analysis, making fault detection more difficult.

Amplitude modulation is an important but seldom understood concept in modeling the forcing function resulting from a fault in the bearing. The effects of amplitude modulation in the frequency domain are summarized by the convolution theorem [5]. Multiplication of the carrier wave by one (or more) modulating signals in the time domain is equivalent to convolution of their Fourier transforms in the frequency domain. The result of this is the presence of sidebands centered around frequencies of the carrier wave. The span of the sidebands is the frequency of the modulating signal.

Modulation models the effect of load fluctuation, which can have two different modes in bearings. The first mode is shaft imbalance, or shaft eccentricity, which causes the entire load profile to be modulated at the shaft rotational frequency. This affects all bearing fault frequencies and causes vibrational energy to move from the impulse harmonics to modulation sidebands around the impulse frequency harmonics. The second mode of modulation is caused when the spall passes in and out of a fixed load zone. This occurs only for faults on the inner (rotating) race or on the rolling elements; a constant radial load will cause any given point on the inner race or rolling element to see a variable load during rotation. This type of modulation affects each fault frequency differently. For spalls on the inner race, the impulse train is modulated by the rotational frequency. For spalls on a rolling element, the impulse train is modulated by the fundamental cage frequency. Spalls on the outer race are unaffected by this phenomenon since they are stationary with respect to the radial load.

\subsection{System Response}

The response of the overall system to the vibration, as seen at a particular sensor location, is predicted by simulating the transmissive paths, illustrated in Figure 2. FEA or hardware ping tests can be used to characterize the transfer function. For a hardware ping test, the full housing is tapped with an impulse hammer, and the resulting vibrations are recorded. Figure 3 shows impact locations and accelerometer locations used for a hardware ping test on one of Sentient Corporation's bearing test rigs. The location of the impacts and the location of the sensors greatly influence the resulting transfer functions.

Using FEA to perform a virtual ping test provides more insight into the mechanisms by which the structure is excited. A stress wave induced by the impulsive load travels through the medium to excite the structure. Figure 4 shows a fast-propagating wave-like deformation of the material around the impact site at approximately 0.00017 seconds after impact. The displacement in the figure has been amplified by 4.5E6 for viewing purposes.

Extensive data from faulted bearing tests performed by Sentient Corporation and by Timken has been used to verify and calibrate the models, as seen in Section 5. 


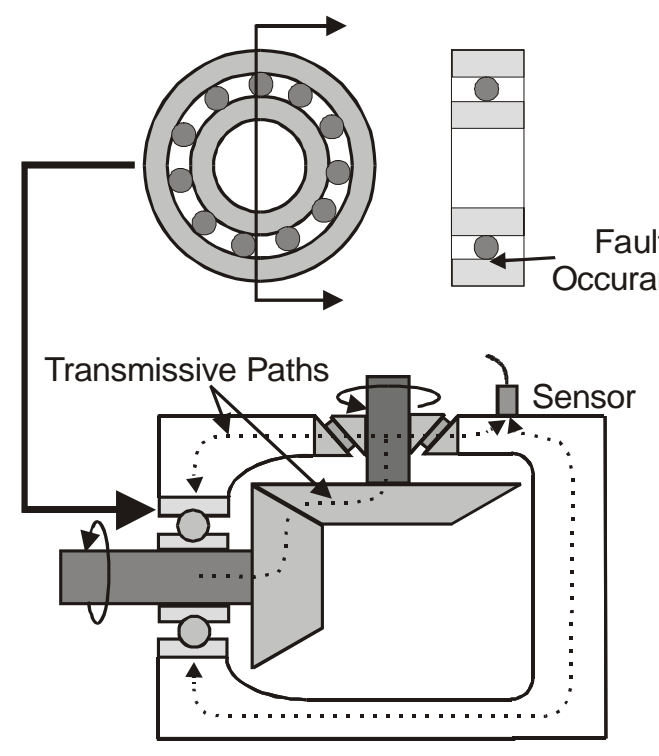

Figure 2 - Illustration of the physical meaning of vibration transmissive paths

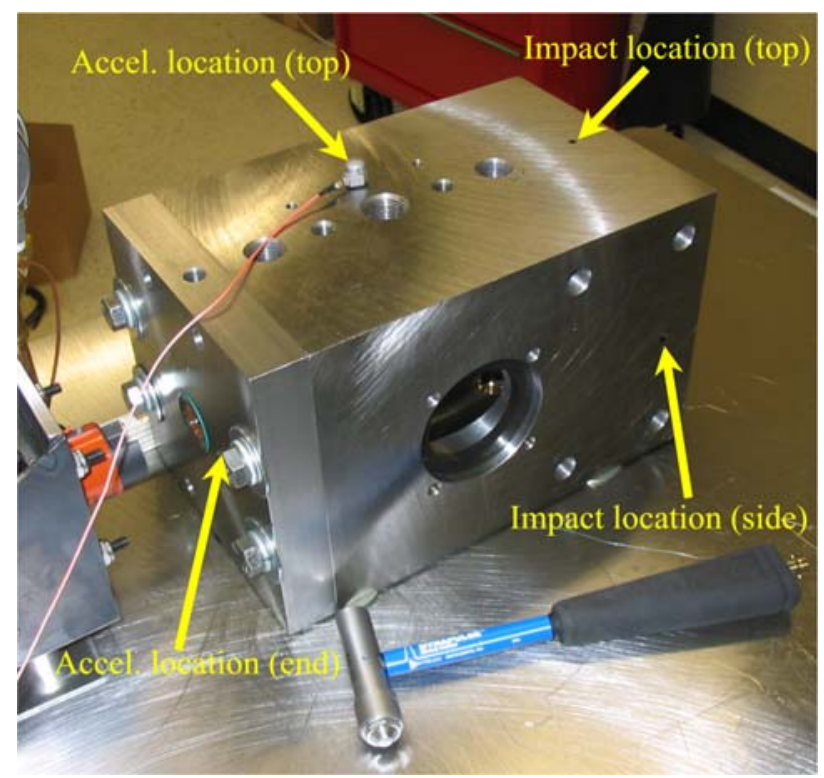

Figure 3 - Bearing test rig housing used for impact tests to determine transfer function

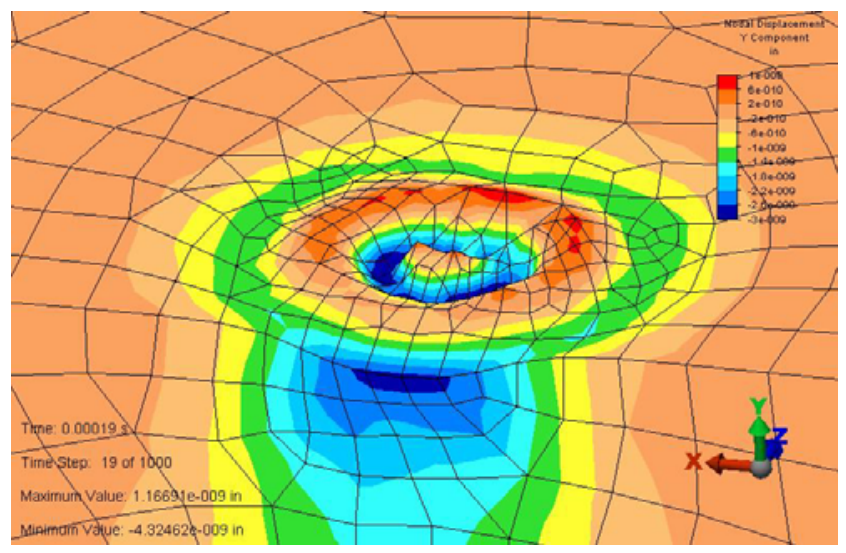

Figure 4 - Fast-propagating wave produced by impact

\section{COMPARISON WITH EXPERIMENTAL RESULTS}

To validate the fault vibration simulator models, output was compared to experimental data.

In the first example, a 106 ball bearing with a small outer race spall and axial loading was run at 2700 RPM in a 2position test rig at Sentient Corporation. Time domain results are shown in Figure 5, with the lower graph showing the results from the model, and the upper graph showing the experimental data. Frequency domain results are shown in Figure 6.

For the second example, a Timken 4-position test rig was used to collect vibration data for a Timken LM501349 tapered roller bearing with large inner race spall and asymmetric loading. Time domain results are shown in Figure 7, and frequency domain results are shown in Figure 8.

The FVS models were set up as follows. Bearing geometry, speed, load, and type of fault were used to generate a realistic forcing function. The forcing function was modulated according to type of loading. Ping tests were used to calibrate the system response.

There are many ways to compare two complex signals: visual inspection of time series or spectral plots; comparison of statistical metrics such as variance; comparison of common diagnostic metrics such as RMS, kurtosis, and crest factor; or even by listening to the signals played through a speaker. It turns out the ear is extremely sensitive to differences in vibration signals, although it cannot usually pinpoint the exact nature of the differences.

Realistic bearing vibration signals must be slowed down approximately 10 times to allow the ear to hear individual 

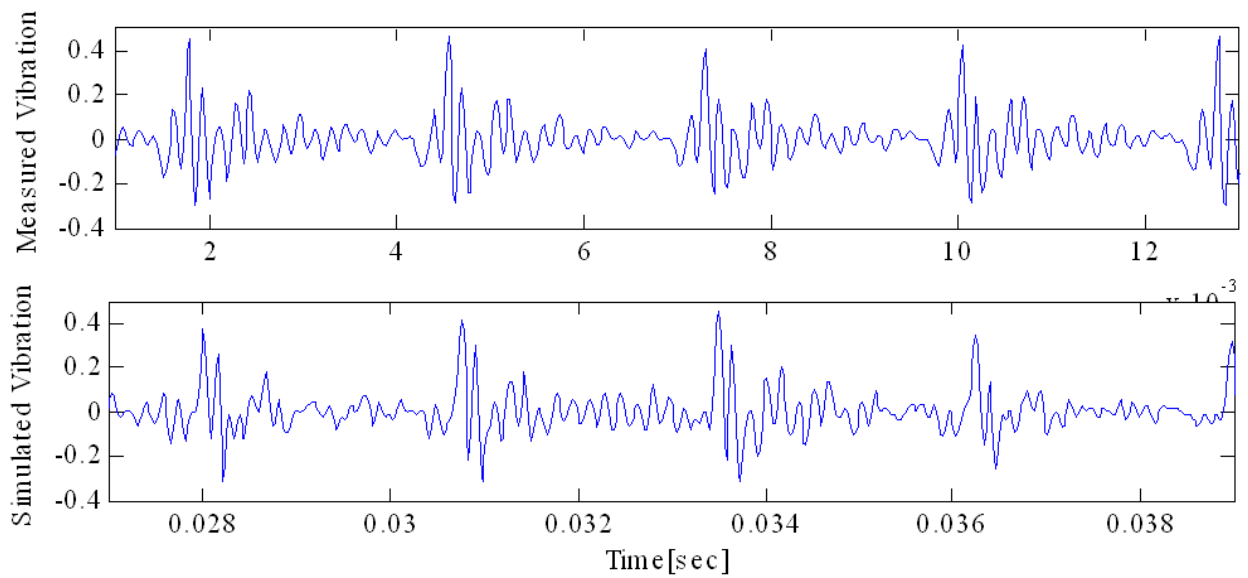

Figure 5 - Time series data for 106 ball bearing

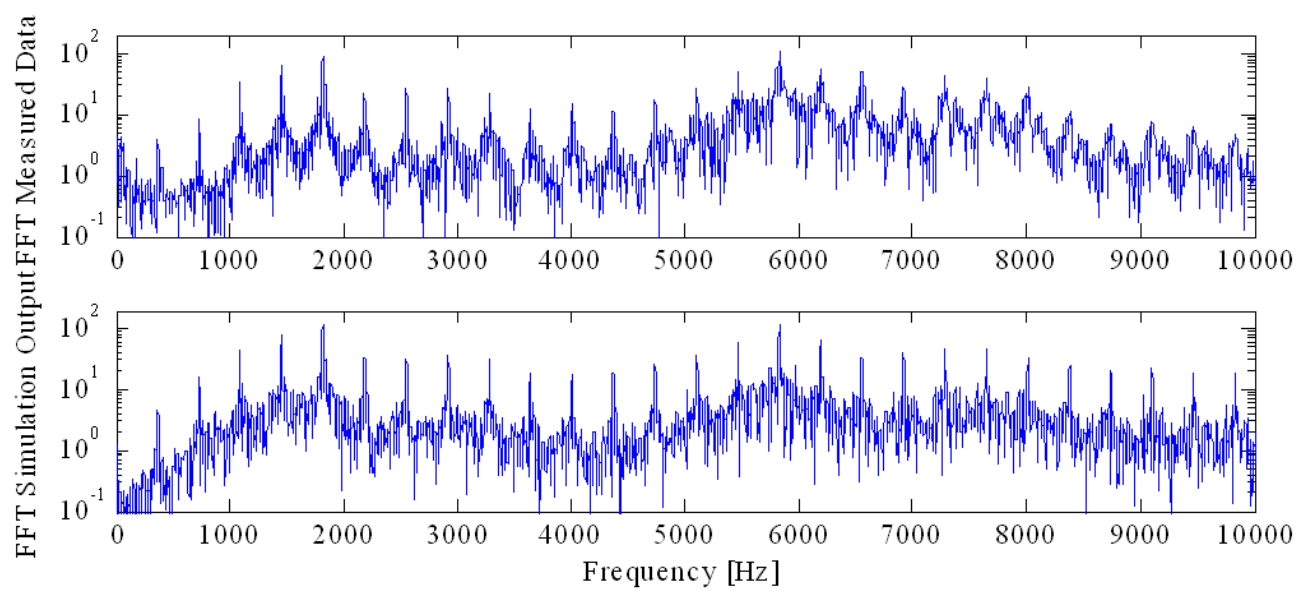

Figure 6 - FFT data for 106 ball bearing

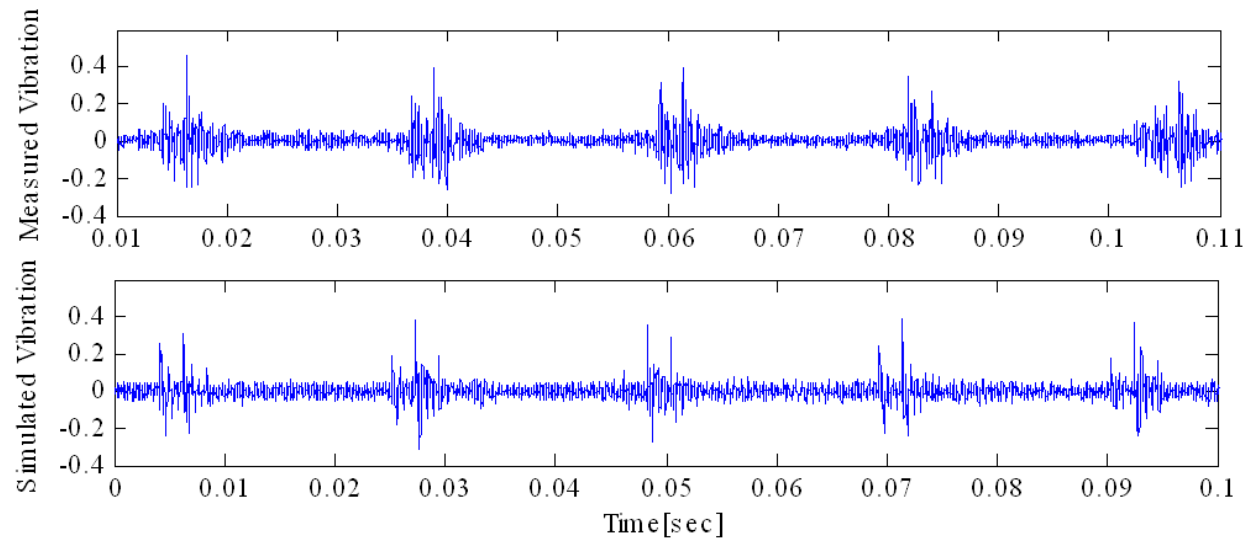

Figure 7 - Time series data for Timken LM501349 tapered roller bearing 


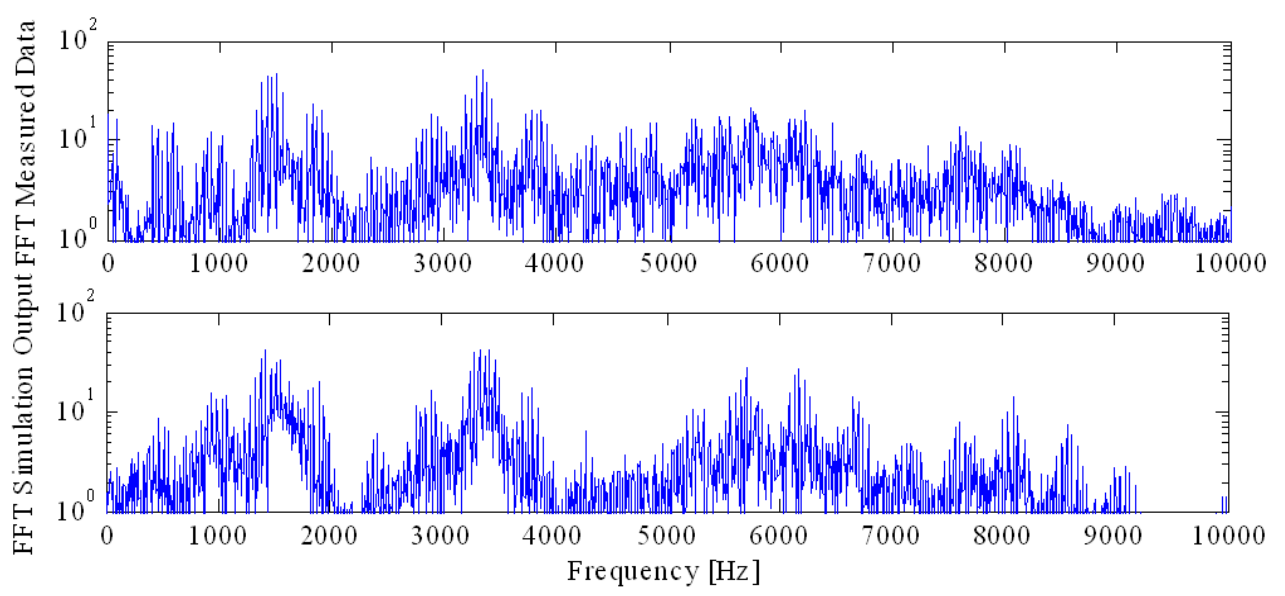

Figure 8 - Frequency data for Timken LM501349 tapered roller bearing

impact events. This is a useful and interesting method for evaluating modeled signal fidelity. However, the most important criterion is whether the model output is interpreted correctly by the standard diagnostics.

Diagnostic comparison metrics for these two examples are shown in Table 1. For the first example, all diagnostic metrics for the simulated signal were within $13 \%$ of the measured signal metric values, and for the second example, all metrics are within $19 \%$. These results indicate that the model does a very good job of duplicating the content of the actual signal.

Table 1 - Diagnostics values for Examples 1 and 2

\begin{tabular}{|c|c|c|c|c|}
\hline & & Measured & Simulated & Difference \\
\hline \multirow{4}{*}{ 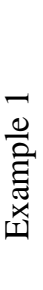 } & RMS & 0.0997 & 0.0931 & $6.69 \%$ \\
\hline & Crest Factor & 4.698 & 5.228 & $11.28 \%$ \\
\hline & Variance & 0.0099 & 0.0087 & $12.93 \%$ \\
\hline & Kurtosis & 7.266 & 7.750 & $6.66 \%$ \\
\hline \multirow{4}{*}{ 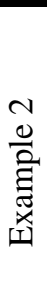 } & RMS & 0.0473 & 0.0425 & $10.04 \%$ \\
\hline & Crest Factor & 9.651 & 9.279 & $3.85 \%$ \\
\hline & Variance & 0.0022 & 0.0018 & $18.89 \%$ \\
\hline & Kurtosis & 14.044 & 15.109 & $7.58 \%$ \\
\hline
\end{tabular}

In all cases tested, there were only small differences in the diagnostic metrics between the real and simulated signals, and the differences were well within the range of variability observed in real signals. In other words, none of the diagnostics examined were able to distinguish the real signal from the simulated signal with any confidence. The only significant difference between the real and simulated signals is greater random variability (through time) of real signals. This will be addressed through the use of stochastic variables.

\section{Applications}

There are many potential uses for the FVS toolset. At the design stage of a new platform or subsystem, the FVS could be used to identify candidate locations for accelerometers and to help develop and test diagnostic and prognostic technologies - all before the hardware has even been built. Once actual hardware is available, the FVS can be calibrated with data from a simple ping test for even greater simulation accuracy. At that point, the output is accurate enough to serve as an evaluation and validation standard for the PHM system: Which algorithm is best at detection of an incipient fault? What is the maximum fault size that ever goes undetected, and under what range of operating conditions? How accurately does the system indicate severity across the operational envelope? By combining the FVS with simple models for other diagnostic indicators (such as debris generation), higher-level fusion and reasoner algorithms can also be validated with realistic data. Expensive operational test data can be leveraged to the fullest extent by running a few critical cases and using the modeling and simulation tools to fill in the gaps.

The FVS would be just as useful for legacy systems. There is currently no standardized methodology for placement of accelerometers. In many cases, diagnostic performance might be improved substantially at little cost by simply relocating sensors. The FVS toolset could be used to identify optimal locations and predict the resulting increase in performance to ensure that the change is worthwhile. Since the FVS simulates normal systemic noise (e.g., gear meshing) as well as fault signals, competing algorithms could also be tested to find solutions with lower false alarm 
rates and better detection capability for a particular application. The model output will be especially accurate where characterization of the hardware is possible.

\section{Conclusions}

The use of PHM technologies is becoming increasingly common. As the aerospace industry evolves from costly time-based maintenance schedules to more efficient condition-based maintenance, the ability to accurately assess the condition and remaining useful life of a monitored component becomes increasingly important. The availability of fault signature data covering the full range of operating conditions is frequently a limiting factor in the development and maturation of PHM technologies.

Vibration data for faulted systems under realistic operating conditions is scarce. Seeded fault tests are costly, and it is often impossible to achieve realistic operating conditions. For these reasons, it is beneficial to develop the technology to accurately simulate fault vibrations for all types of rotating components.

Comparisons on several different machines, using both roller and ball bearings, under a range of loads and speeds, demonstrated that the fault vibration simulator works well across a broad application spectrum. The modular architecture is applicable to all types of rotating components. The model is highly developed for bearings and has been calibrated for several types. Calibration for gears is in process. Results demonstrate an excellent correspondence between simulated and actual signals, with all important diagnostic features matching. Much knowledge and insight has been gained into the sources of mechanical vibration and modes of transmission in typical aerospace systems.

\section{REFERENCES}

[1] P. D. McFadden and J. D. Smith, "Model for the Vibration Produced by a Single Point Defect in a Rolling Element Bearing," Journal of Sound and Vibration 96(1), 69-82, 1984.

[2] P. D. McFadden, "Detecting Fatigue Cracks in Gears by Amplitude and Phase Demodulation of the Meshing Vibration," Journal of Vibration, Acoustics, Stress, and Reliability in Design 108,165-170, April 1986.

[3] J. I. Taylor, "Identification of Bearing Defects by Spectral Analysis,” Journal of Mechanical Design 102, 199-204.

[4] Mitchell Leobold et al., "Review of Vibration Analysis Methods for Gearbox Diagnostics and Prognostics," Proceedings of the $54^{\text {th }}$ Meeting of the Society for Machinery Failure Prevention Technology, May 1-4, 2000, 623-634.

[5] Richard G. Lyons, Understanding Digital Signal Processing, Massachusetts: Addison Wesley, 1997.

\section{ACKNOWLEDGMENTS}

Portions of this research were funded under Navy SBIR topic \# N04-028. Sentient Corporation would like to thank the JSF program office and NAVAIR for their support.

\section{BIOGRAPHY}

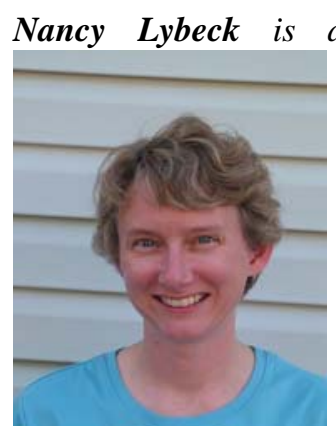

a Research Scientist at Sentient Corporation. She holds a PhD in Mathematics from Montana State University. She has developed algorithms for a variety of applications, including modelbased diagnostics, data reduction algorithms for turbine engine usage data, and models of dynamic response for engineering materials.

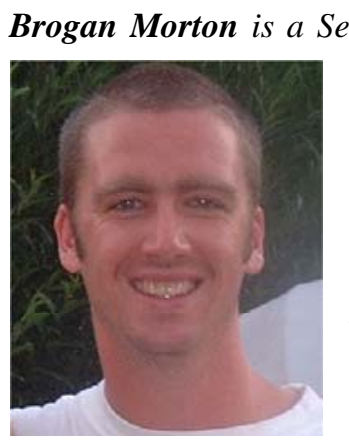
Corporation and serves as the discipline lead in vibration diagnostics. He is also the lead developer for the Fault Vibration Simulator. He holds a Master of Science in Mechanical Engineering from the University of New Hampshire.

Sean Marble is the President of Sentient Corporation. He

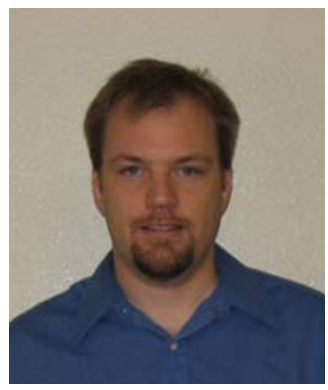
holds a Master of Science in Mechanical Engineering from Purdue University. He has researched the physics of machine component failure and developed diagnostic and prognostic technologies for aerospace and industrial applications for over 10 years.

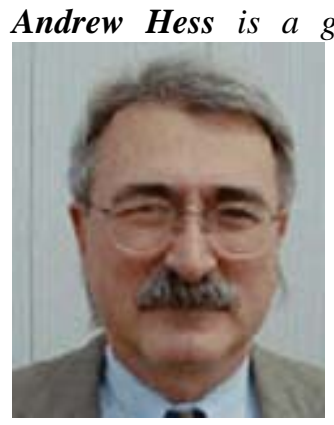

graduate of both the University of Virginia and the U. S. Navy Test Pilot School. Andy has been a leading advocate for health monitoring in the Navy and has been instrumental in the development of every Navy aircraft application through the F/A-18 and $\mathrm{V}$-22. His efforts have largely led to the successful transition of the IMD HUMS for all $\mathrm{H}-60, \mathrm{H}-53$, and $\mathrm{AH}$ 1 aircraft. He is currently Lead Development Manager for the JSF PHM system. 
John Kelly is an Electronics Engineer at the Patuxent River

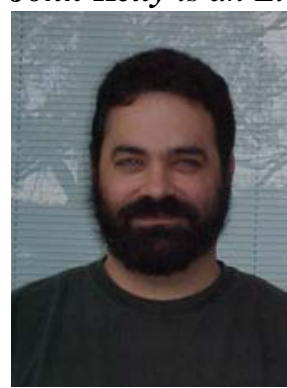

Naval Air Warfare Center - Aircraft Division. He earned BSAE and BSEE degrees from Parks College of St. Louis University and a MSEE from Southern Illinois University at Edwardsville. He has been involved in Integrated Diagnostics analysis programs for multiple NAVAIR and joint service platforms. 